\title{
EDITORIAL
}

\section{Cardiorenal Syndrome: Difficult Treatment Decisions}

Acute or chronic dysfunction of the heart or kidneys can induce acute or chronic dysfunction in the other organ. In addition, both heart and kidney function can be impaired by an acute or chronic systemic disorder. The term "cardiorenal syndrome" (CRS) has been applied to these interactions. In type $1 \mathrm{CRS}$, acute heart failure (HF) leads to worsening kidney function. In type $2 \mathrm{CRS}$, chronic HF causes progressive chronic kidney disease. A 2004 report from the National Heart, Lung, and Blood Institute defined CRS as a condition in which therapy to relieve congestive symptoms of HF is limited by a decline in renal function as manifested by a reduction in GFR. The reduction in GFR was initially thought to result from a reduction in renal blood flow. However, various studies have demonstrated that cardiorenal interactions occur in both directions and by a variety of mechanisms. We have many patients admitted in CCU with CRS in hospital practice

The prevalence of moderate to severe kidney impairment (defined as a glomerular filtration rate [GFR] less than $60 \mathrm{~mL} / \mathrm{min}$ per $1.73 \mathrm{~m}^{2}$ ) is approximately 30 to 60 percent in patients with HF. In addition to these baseline observations, patients undergoing treatment for acute or chronic HF frequently develop an increase in serum creatinine, which fulfills criteria for type 1 or type 2 CRS. Chronic heart failure affects approximately $1-2 \%$ of the adult population in developed countries, with increasing prevalence $(\geq 10 \%)$ in patients over the age of 70 years. Renal dysfunction is common in patients with CHF. One study found that $33 \%$ of CHF patients with New York Heart Association (NYHA) class III and IV symptoms had severely impaired renal function (creatinine clearance $<30 \mathrm{ml} / \mathrm{min}$ ).

Reduced glomerular filtration rates (GFR) are common in patients presenting with heart failure (HF) and are associated with increased mortality. A systematic review found that mortality increased by approximately 15 percent for every $10 \mathrm{~mL} / \mathrm{min}$ reduction in estimated GFR.

Renal function (eGFR) and serum potassium should be checked before initiating ACEI, ARB or MRA therapy, and repeated after 1-2 weeks of treatment and after each change in dose, with routine monitoring every 4 months in stable patients. Caution or specialist advice' is advised in patients who have significant renal dysfunction (defined as a creatinine level of $\geq 2211 / 4 \mathrm{~mol} / 1$ or estimated glomerular filtration rate [eGFR] $<30 \mathrm{ml} / \mathrm{min} / 1.73 \mathrm{~m} 2$ CKD stage IV), Combined hydralazine and isosorbide dinitrate is an alternative first-line treatment for those who are unable to tolerate an ACEI or ARB, but this combination does not directly address ongoing albuminuria, neither does it necessarily prevent further decline in renal function.

If serum potassium is $>5.0 \mathrm{mmol} / \mathrm{l}$, an ACEI, ARB or MRA should not be started immediately. A modest decline in renal function can be expected upon initiation of treatment with an ACEI, ARB or MRA as the result of haemodynamic reduction in intraglomerular pressure for expected changes in renal function. In these circumstances, the dose should not be modified but the renal function rechecked in 2 weeks.

According to ESC with the introduction of ACEI/ARB up to $50 \%$ increase in creatinine, or creatinine to 266 $1 / 4 \mathrm{~mol} / 1$ (eGFR $<25 \mathrm{ml} / \mathrm{min} / 1.73 \mathrm{~m} 2$ ), whichever is smaller, is acceptable. With the introduction of MRA, creatinine rise to e" $221 \frac{1}{1} / 4 \mathrm{~mol} / 1$ or eGFR $<30 \mathrm{ml} / \mathrm{min} /$ $1.73 \mathrm{~m} 2$ indicates that the dose/ should be halved. With a creatinine rise to $>3101 / 4 \mathrm{~mol} / 1$ or eGFR $<20 \mathrm{ml} / \mathrm{min} /$ $1.73 \mathrm{~m} 2$, MRA should be stopped. According to NICE guidelines with the introduction of ACEI/ARB $<25 \%$ decrease in eGFR or $<30 \%$ increase in creatinine is acceptable. Acidosis is common in CKD. It can hasten long-term decline in glomerular filtration rate and exacerbate hyperkalaemia. So use of sodium bicarbonate (500-1000 mg twice daily) can help temper hyperkalaemia, without significant adverse effect.

However, other evidence suggests that patient outcomes may be improved with aggressive fluid removal even if accompanied by a rise in serum creatinine. Many of the medications used in CHF are themselves potentially nephrotoxic, mandating careful collaboration between cardiology and nephrology teams Given the limitations imposed by impaired renal function on the ability to correct volume overload and the strong association 
between impaired or worsening renal function and adverse clinical outcomes in patients with $\mathrm{HF}$, it is possible that effective treatment of the cardiorenal syndrome (CRS) would improve patient outcomes. On the other hand, the worse prognosis associated with CRS could primarily reflect a reduced GFR being a marker of more severe cardiac disease. In this setting, improving renal function alone would not necessarily improve patient outcomes.

The effect of diuretic-induced fluid removal on the GFR is variable in patients with HF. Although fluid removal may result in increases in serum creatinine and rising serum creatinine is associated with worse prognosis in patients with HF, aggressive decongestion leading to worsening renal function may be associated with improved survival. There is increasing interest in the role of ultrafiltration and peritoneal dialysis in the management of acute and chronic heart failure, respectively, but outcome evidence is currently lacking. Evidence from the UNLOAD trial suggests that the selected use of ultrafiltration may safely enable greater fluid loss than can be achieved using intravenous diuretics, with fewer readmissions due to fluid reaccumulation at 90 days. One small study of 17 patients with refractory congestive heart failure suggested that peritoneal dialysis improved NYHA functional class at 1 year, as well as reducing hospital admissions

Mortality is increased in patients with heart failure (HF) who have a reduced GFR. The majority of patients who have advanced chronic kidney disease (CKD) will die from cardiac causes; this may be even more common than reaching dialysis. Conversely, patients with cardiac disease who have concomitant CKD have poorer outcomes. Patients with chronic kidney disease (CKD) have an increased risk of both atherosclerotic cardiovascular disease and HF, and cardiovascular disease is responsible for up to 50 percent of deaths in patients with renal failure Acute or chronic systemic disorders can cause both cardiac and renal dysfunction.

For the primary prevention of CRS, lifestyle measures, such as increasing physical activity, smoking cessation, healthy diet, weight loss and blood pressure and cholesterol control are equally important in both renal and cardiac disease, which share many common underlying comorbidites, including age, hypertension, dyslipidaemia, smoking and diabetes mellitus.

\section{Dr. Manzoor Mahmood}

Associate Professor of Cardiology, Bangabandhu Sheikh Mujib Medical University (BSMMU), Shahbag, Dhaka, Bangladesh.

\section{Prof. Harisul Hoque}

Professor of Cardiology and Head of Heart Failure, Cardiac Rehabilitation and Preventive Cardiology Division, BSMMU, Dhaka.

\section{References:}

1. Goldsmith D and Scully P. The managament of end-stage heart failure and reducing the risk of cardiorenal syndrome. Clinical Medicine 2013. Volume 13, No-6:610-13

2. Keiman MS, Udelson JE, Samak M et al. Cardiorenal Syndrome: Prognosis and Management 2018 https://www.uptodate.com/ contents/cardiorenal-syndrome-prognosis-and-treatment (accessed online 27-06-2018) 